\title{
Surgical repair of paralytic lagophthalmos by medial tarsal suspension of the lower lid
}

\author{
María Castroviejo-Bolibar, Antonio de Damborenea, Alvaro Fernández-Vega
}

\begin{abstract}
Background-The seventh nerve palsy causes loss of function in the affected orbicularis oculi muscle. There is exposure and dessication of the corneal surface and obvious aesthetic changes in facial symmetry.

Method-A surgical procedure has been used in 11 patients to substantially improve palpebral closure. The technique consists of a small upper lid tarsoconjunctival flap sutured into a nasal pretarsal pocket of the lower lid, achieving a medial tarsal suspension of the lower lid.

Results-This technique improves cosmesis, is reversible, and combinable with other procedures. The signs and symptoms of exposure keratitis are satisfactorily resolved. There have been no complications in an average follow up of $51 / 2$ years after this surgical procedure. Conclusion-This technique could be considered an appropriate treatment for patients suffering temporary or permanent facial palsy.

(Br F Ophthalmol 1996;80:708-712)
\end{abstract}

The paralytic ectropion pathogenetic mechanism is similar to the complete laxity of orbicularis oculi muscle with lid retraction, and lagophthalmos is often the rule. The lower third of the cornea is the most affected area. In those cases in which patients lose Bell's phenomenon, all the corneal surface is unprotected even with forced closure. An annoying complication is epiphora. There is irritation in spite of tearing because blinking is disturbed and the lacrimal gland may also be affected. Involvement of the greater superficial petrosal nerve impairs reflex tear secretion. The atonic ectropion needs surgical repair ${ }^{1}$ and in the case of keratopathy, surgery must be done as soon as possible. Eyelid paralysis creates functional deficits which may lead to blindness. Thus, management of paralysis following facial nerve injury is extremely important. ${ }^{2}$

Eye Bank of the Red

Cross of Vizcaya,

Spain

A de Damborenea

Ophthalmological Clinic, Oviedo, Spain

A Fernández-Vega

Correspondence to: Dr María Castroviejo, Apartado de Correos 762, 15700 Santiago de Compostela, Spain. exposure keratopathy. The purpose, and risks of the procedure were explained to the patients. The main goal was to obtain ocular protection and thereby the elimination of exposure keratopathy. Secondary goals in- cluded the elimination of epiphora, ocular pain and pruritus, satisfactory cosmesis, and minimisation of the use of topical lubricants. The consistency of the technique and the possibility of its combination with other surgical procedures were considered. A careful history was taken and clinical manifestations were recorded and graded (from 0 to 4 ) preoperatively and then postoperatively every month for the first 3 months, then every 3-6 months for the first year, and thereafter every 6-12 months. All results were registered except for case 8 .

\section{Surgical procedure (Figs 1 and 2)}

Local anaesthesia was used with $1 \%$ lignocaine with 1:200 000 adrenaline, 10 minutes before the start of the operation. To prepare the flap of the upper lid it is first everted. A nasal incision perpendicular to and $2-3 \mathrm{~mm}$ above the lid margin is made in the tarsus. A second incision is made through the tarsus parallel to the former, to create a tarsoconjunctival flap (TCF) $4 \mathrm{~mm}$ wide and $8 \mathrm{~mm}$ high, just temporal to the punctum. Then, a third incision parallel to and $2-3 \mathrm{~mm}$ above the lid margin is carried out, joining the two vertical incisions. These do not involve the pretarsal region of the orbicularis muscle. The entire flap is freely dissected with the exception of a superior base (Fig 1a). The TCF can be lengthened by dissecting Müller's muscle above the superior tarsal border. The conjunctiva of the flap is removed by curettage. The TCF can be also obtained in a similar fashion from the lateral third of the upper lid to achieve a temporal tarsoconjunctival flap.

The lower lid pocket should be fashioned slightly broader than the TCF and $1-2 \mathrm{~mm}$ temporal to the punctum. The incision into the pocket can be created in one of two ways-a transmarginal incision through the grey line or a horizontal incision through the tarsal surface of the lower lid. The pocket is deepened between the plane of the tarsus at the back and the pretarsal orbicularis in front (Fig 1b).

A 5-0 doubled armed silk suture is used to attach the TCF to the lower lid. A ' $U$ ' suture, with the base of the ' $U$ ' horizontal is placed through the tarsus of the TCF. Both arms of the suture are then passed freely within the pocket and pulled out through the skin. The pulling motion then inserts the TCF from the upper lid into the lower lid pocket. The suture is knotted on a bolster and removed in 10 to 15 days (Fig 1 c, d).

In the case of recovery of the facial function the TCF can be severed at the lower lid margin. This procedure is best performed over 


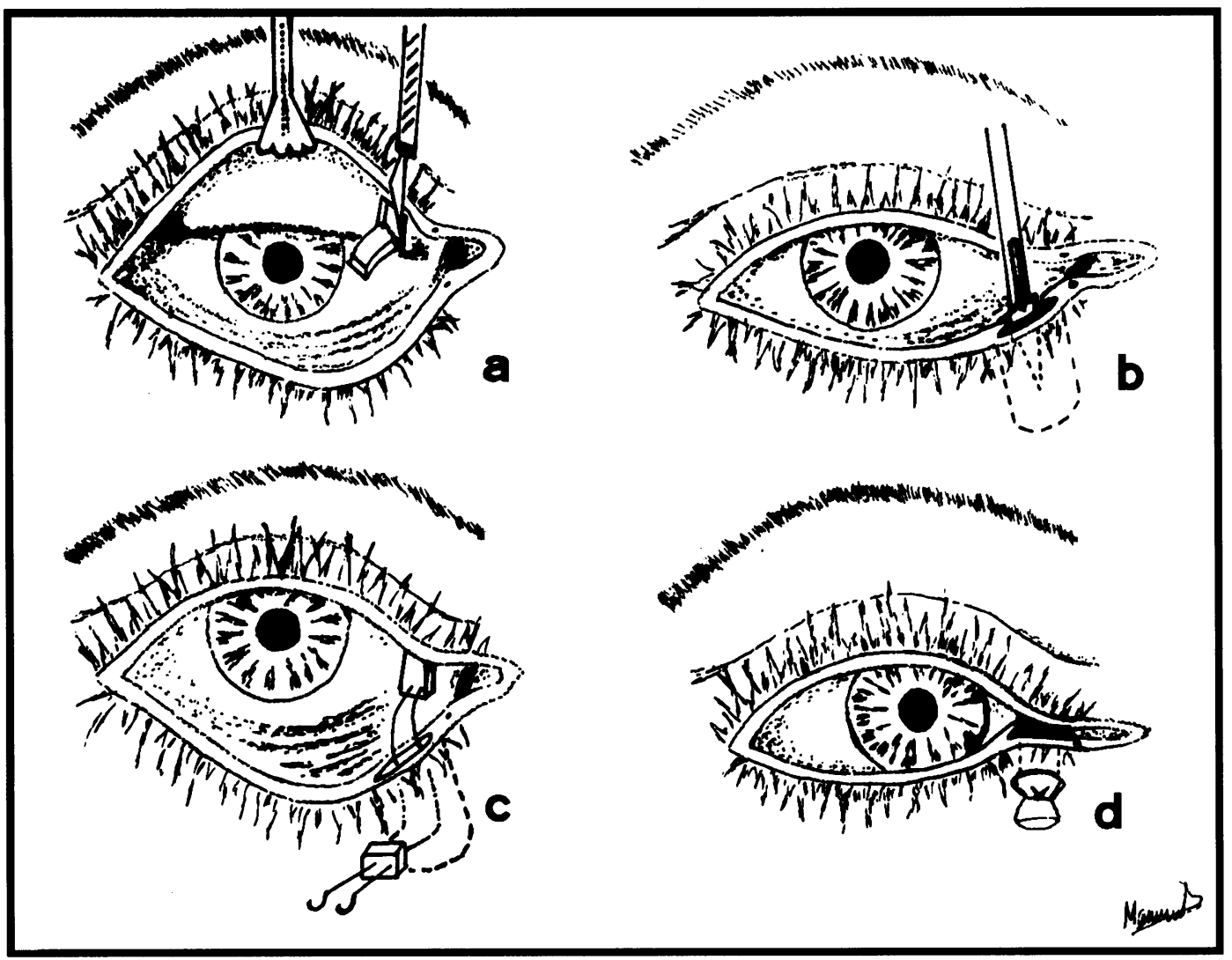

Figure 1 Preparation of the upper lid tarsoconjunctival flap (TCF) and the lower lid pocket. (a) The upper lid is everted and a nasal TCF is dissected. (b) For the lower lid pocket a horizontal incision is made through the grey line of the lid margin. The pocket is deepened between the plane of the tarsus and the orbicularis. (c) $A$ ' $U$ ' suture, with the loop horizontal is passed through the tarsus of the tarsoconjunctival flap. The needles cross the pocket freely and then are pulled through the skin inserting the flap inside the pocket. (d) The suture is knotted on a bolster.

a protective instrument. Since the flap retracts into the upper lid, it does not need to be sutured.

\section{Case reports}

CASE 1

A 72-year-old man had been suffering from a right facial palsy for 6 years after an operation on the parotid gland. A tarsorrhaphy was performed elsewhere, causing deformity of the lower lid margin. He had chronic keratopathy, lower lid retraction, punctal eversion, and lagophthalmos of the right eye (Fig 3). A medial tarsal suspension (MTS) was performed as described above. The patient's signs and symptoms have been resolved for $61 / 2$ years.

\section{CASE 9}

An 82-year-old woman had a tumour of the left lower lid resected 7 years ago. The area of excision included the lower half of the inner canthus, the lower lacrimal drainage system, and the medial half of the lower lid. The medial canthal and nasal lid areas were restored by a full thickness skin graft and a sliding flap from the lateral eyelid and the temporofacial region. A temporal tarsal suspension was performed in order to reconstruct the lateral canthus. No defect could be found in the lid closure, and no relapse in 7 years. The cosmesis was very satisfactory (Fig 4).

\section{Results}

The MTS technique proved to be effective and safe. The mean postoperative follow up period was 5 years and 6 months, ranging from 11
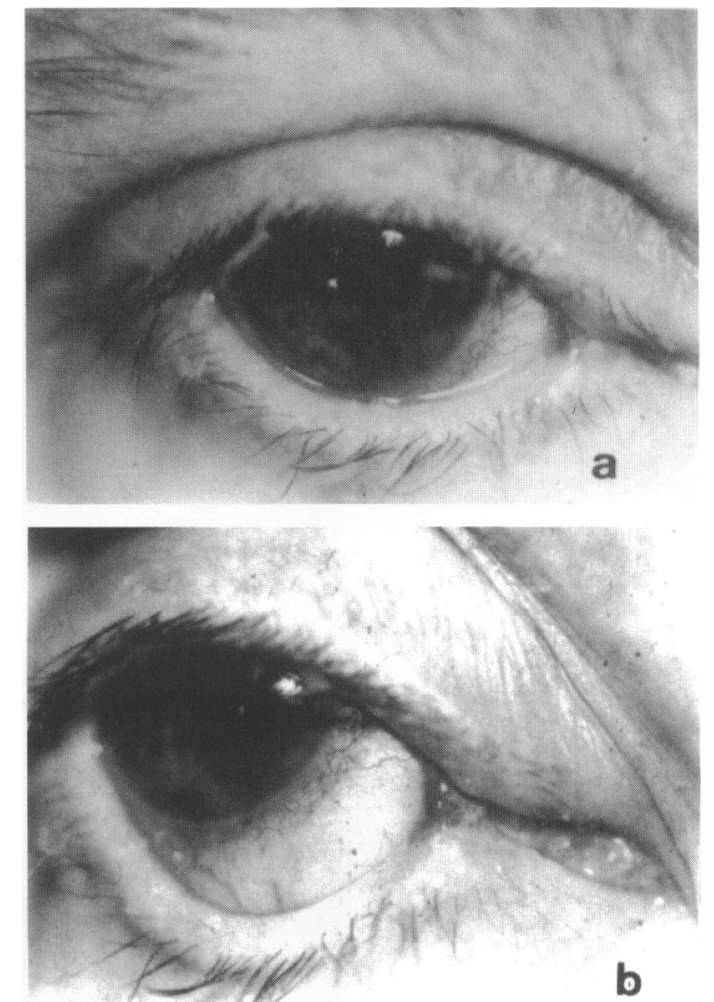

Figure 2 (a) Appearance 2 weeks after the medial tarsal suspension of the lower lid. In normal conditions the flap is barely visible. (b) It can be better seen when the lower lid is pulled down.

months to $81 / 2$ years (Table 1 ). The MTS improved lid-globe apposition and facilitated lacrimal drainage. Ectropion was successfully corrected in all cases. Epiphora decreased in all 


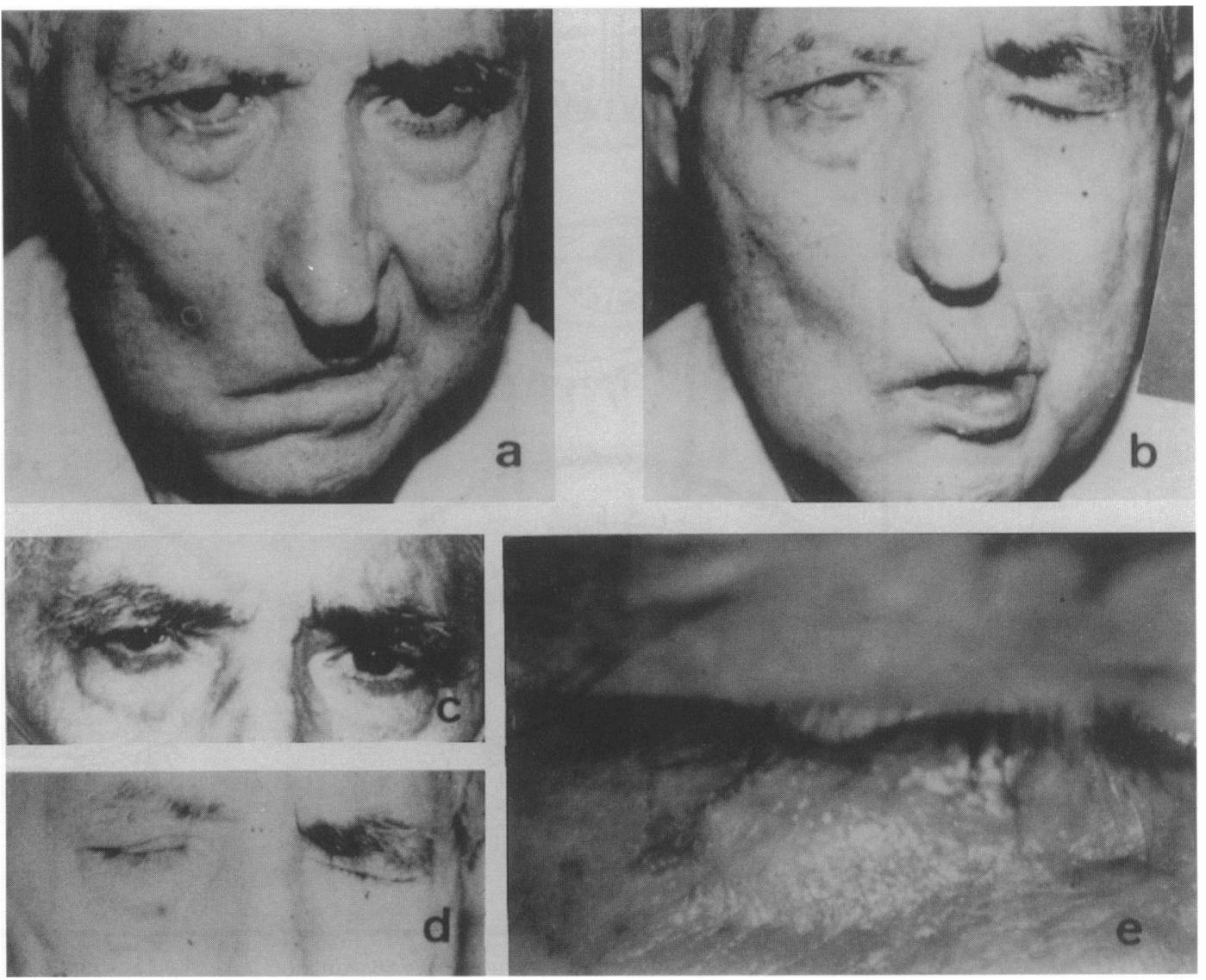

Figure 3(a,b) Ectropion and lagophthalmos before surgery. (c,d) 10 days after medial tarsal suspension an improvement in cosmesis and function is obvious. (e) When closure is not forced, the deformity of the lower lid margin is noticeable because of a previous tarsorrhaphy.
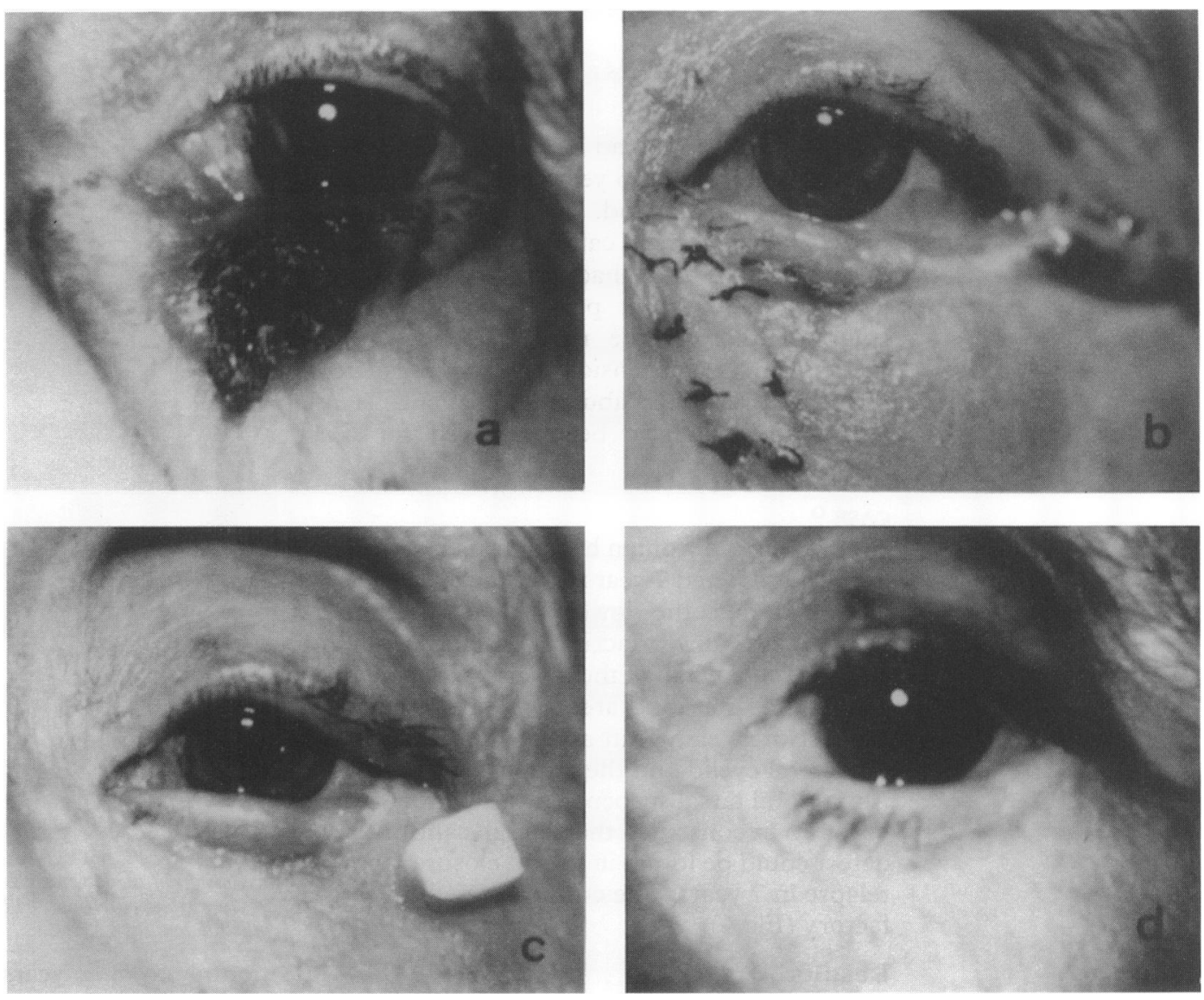

Figure 4 Basal cell carcinoma affecting the lower eyelid and caruncula. (a) The black marks correspond to the limits of the skin resection. (b) Firstly, the nasal canthus was reconstructed. (c) Two weeks after the excision a temporal tarsal suspension of the lower lid was performed to correct the lateral lagophthalmos. (d) Final appeareance. 
Table 1 Postoperative follow up time and surgical assessment of medial tarsal suspension (MTS) of the lower lid

\begin{tabular}{lllll}
\hline Patient No & Length of follow up & Previous surgery & $\begin{array}{l}\text { Combined surgery } \\
\text { at MTS operationt }\end{array}$ & $\begin{array}{l}\text { Further surgery } \\
\text { neededf }\end{array}$ \\
\hline 1 & 6.6 years & Yes (a) & No & No \\
2 & 7.6 years & No & No & No \\
3 & 1.3 months & No & No & No \\
4 & 8.6 years & No & Yes (i) & No \\
5 & 8 years & No & No & No \\
6 & 5 years & Yes (b) & Yes (ii) & Yes (I) \\
7 & 5.9 years & No & Yes (iii) & No \\
8 & 7 & No & No & No \\
9 & 7 years & Yes (c) & No & No \\
10 & 5 years & Yes (d) & No & Yes (I) \\
11 & 11 months & Yes (e) & No & \\
\hline
\end{tabular}

^ (a) Tarsorrhaphy; (b) encircling fascia; (c) basal cell carcinoma; (d) facial nerve decompression (e) maxillary sinus carcinoma.

† (i) Lateral canthoplasty; (ii) fascial extraction; (iii) block resection of the lower lid.

$\ddagger$ (I) For slight lagophthalmos.

Table 2 Summary of subjective and objective clinical findings after medial tarsal suspension

\begin{tabular}{|c|c|c|c|c|c|}
\hline \multirow[b]{2}{*}{ Patient No } & \multirow[b]{2}{*}{ Epiphora } & \multirow[b]{2}{*}{ Ocular pain or itching } & \multicolumn{2}{|c|}{ Cosmetic evaluation } & \multirow[b]{2}{*}{ Exposure } \\
\hline & & & Patients & Doctors & \\
\hline 1 & 4 & 4 & 4 & 4 & $3+$ \\
\hline 2 & 4 & 4 & 4 & 4 & 4 \\
\hline 3 & 3 & 4 & 4 & 3 & 4 \\
\hline 4 & 4 & 4 & 4 & 4 & 4 \\
\hline 5 & 3 & 4 & 4 & 4 & 4 \\
\hline 6 & 3 & 2 & 3 & 3 & $3 t$ \\
\hline 7 & 4 & 4 & 4 & 4 & 4 \\
\hline 8 & - & - & - & - & - \\
\hline 9 & $1 \star$ & 4 & 4 & 4 & 4 \\
\hline 10 & 2 & 4 & 3 & 3 & 4 \\
\hline 11 & 4 & 4 & 4 & 3 & $3 t$ \\
\hline
\end{tabular}

Grades: 0 =worse; 1 =no improvement; $2=$ =satisfactory; 3 =good; $4=$ =xcellent.

* Excision of the lower lacrimal drainage system.

† Conjunctival exposure (see text). that the rim of the tarsus must be preserved to prevent postoperative eyelid malposition. Smith and de Damborenea reported this procedure using a lateral tarsoconjunctival pillar (partial preliminary results were presented by Smith BC and de Damborenea $A$ at the 19th Annual Scientific Symposium of the American Society of Ophthalmic Plastic and Reconstructive Surgery, Las Vegas, 1988).

We now prefer the upper lid flap to be medial, instead of temporal, to ensure close contact between the inferior punctum and the eye globe. This improves ephiphora and patient comfort. On the other hand, we have not seen postoperative lower lid eversion, trichiasis, wound dehiscences, or granuloma formation as it was reported by other authors with the tarsal pillar technique. ${ }^{5}$ The preparation of the lower lid recipient bed and the sutured closure in their technique differs from our procedure. We believe that the lower lid pocket in the MTS procedure has several advantages. It contributes to lower lid stability by evenly distributing the suspensory strength of the TCF and obtains a good lid-globe apposition. Because it provides a larger contact area it secures the adherence between the flap and the lower lid. This adhesion is further enhanced by removing the conjunctiva of the flap. This tight adhesion could explain our lack of wound dehiscences, even in the case of the patient who pulled out the suture on the third postoperative day. A third advantage of the lower lid pocket is that the free border of the flap is hidden and buried when it is sutured, minimising granuloma formation. According to our study, the MTS procedure proved to be effective during an average follow up of nearly 6 years and therefore can be considered a permanent, yet reversible, technique to diminish the interpalpebral fissure. The MTS is relatively easy to manage and quick to perform when compared with other procedures. ${ }^{267}$ Since it is also readily reversible, if the function of the seventh nerve is recovered, the tarsoconjunctival flap simply has to be severed. This is performed with minimal deformities. If postoperatively the patient is undercorrected, further lid closure may be achieved by performing a temporal tarsal suspension of the lower lid, or a tarsal strip canthoplasty, to tighten and elevate the paralysed eyelid. Moreover, the MTS technique preserves the majority of the lid and tarsus, permitting it to be combined with techniques such as gold weight implantation. As can be seen in case 9, the tarsal suspension procedure is also useful in correcting certain cases of palpebral defects with symptomatic lagophthalmos.

Many procedures have been described to improve lid closure of paralytic lagophthalmos but none of them has proved helpful in all cases for extended periods of time. ${ }^{27-9}$ The MTS technique has produced great improvement in all patients and may achieve a successful long term result as we have shown in this study.

The authors are grateful to Dr Byron C Smith for his kind collaboration in some of the operations described above. We would like to dedicate this paper to his memory. responsible for lid margin tears and deformities. We have not observed complications such as entropion, trichiasis, or deformities of the upper lid because $2-3 \mathrm{~mm}$ of the lid margin remain untouched. Smith et $a l^{34}$ emphasised 
1 Duke-Elder S. Diseases of the eyelids. In: System of ophthalmology. 1 st ed. London: Henry Kimpton, 1974

2 Gilbard SM, Daspit CP. Reanimation of the paretic eyelid using gold weight implantation. Ophthalmic Plast Reconst Surg 1991;7:93-103.

3 Smith B, Cherubini TD. Oculoplastic surgery. St Louis: CV Mosby, 1970:32.

4 Smith B, Nesi FA. Practical techniques in ophthalmic plastic surgery. St Louis: CV Mosby, 1981:74.

5 Tanenbaum M, Gossman MD, Bergin DJ, Friedman HI Lett D, Haines $\mathrm{P}$, et al. The tarsal pillar technique for the narrowing and maintenance of the interpalpebral fissure. Ophthalmic Surg 1992;23:418-25.

6 Leatherbarrow B, Collin JR. Eyelid surgery in facial palsy. Eye 1991;5:585-90.

Sullivan TJ, Collin JRO. Medial canthal resection: an effective long-term cure for medial ectropion. $\mathrm{Br} \mathcal{F}$ Ophthalmol 1991;75:288-91.

$8 \mathrm{McCord}$ CD Jr. Surgery of the eyelids. In: Duane TD, ed. Clinical ophthalmology. Philadelphia: JB Lippincott, 1988:28.

9 Lisman RD, Smith B, Baker D, Arthurs B. Efficacy of surgical treatment for paralytic ectropion. Ophthalmology 1987;94:671-81. 